\title{
Therapeutic Effect of Allicin on Glioblastoma
}

\author{
Cheng Wang* \\ Department of Neurosurgery, The Second Affiliated Hospital of Zhejiang Chinese Medical University, China
}

*Corresponding author: Cheng Wang, Department of Neurosurgery, The Second

Affiliated Hospital of Zhejiang Chinese Medical University, China.

Received Date: March 12, 2020

Published Date: April 28, 2020

\begin{abstract}
Glioblastoma (GBM), as a common malignant tumor of central nervous system, is lack of effective treatments. Most of the GBM patients die in two years. Lack of effective chemotherapy is a important reason for the poor therapeutic efficacy of GBM. Several natural dietary agents have established noteworthy consideration because of their pharmacological effects. Garlic is one of the earliest cultivated plants and is commonly used as a dietary condiment worldwide. Alliin, as the most important active substance of garlic, has been proved to have diverse biologic activities including anti-inflammatory effects, antitumor effects and other effects. This article briefly reviews the therapeutic effects and mechanisms of allicin and its metabolites on GBM.
\end{abstract}

Keywords: Glioblastoma; Garlic; Alliin; Apoptosis; ROS; Histone Deacetylase

\section{Introduction}

Glioblastoma (GBM) are the most common central nervous system malignant tumor in clinic, accounting for $46.6 \%$ of all primary central nervous system tumors [1]. GBM is characterized by high degree of malignancy, strong invasion, and easy recurrence. The current standard of care for GBM begins with maximal safe surgical resection followed by a combination of radiotherapy (RT) with temozolomide therapy [2]. Adjuvant therapy can significantly prolong the median survival of GBM patients and improve their life quality, but still cannot achieve desired effect. The median survival for patients with GBM tumors is only 12-18 months and the majority of these patients die in two years [3]. Garlic, a member of family liliaceae and originally from central Asia, is one of the earliest cultivated plants [4]. Garlic is a dual-purpose plant for medicine and food. It has been used as a traditional chinese medicine for thousands years. It contains a variety of chemical components, including sulfur compounds and saponins. Alliin, as the main sulfide, is the most important active substance. After processing, such as cutting, crushing, chewing, or dehydration, alliinase rapidly breaks down alliin to form allicin. Allicin is immediately decomposed to other organosulfur compounds such as diallyl sulphide (DAS), diallyl disulfide(DADS), and diallyl trisulfide (DATS), dithiins, and ajoene [5]. In previous studies, allicin and its metabolites have been proved to have diverse biologic activities including antiinflammatory effects, antitumor effects and other effects [6].

\section{Therapeutic Effect of Allicin on GBM}

\section{Allicin inhibits proliferation and viability in glioma cells}

Inhibition of cell viability and proliferation is an important part of glioma treatment. Alliin has been revealed of dose-dependent inhibition of proliferation and viability of U251 glioma cells [7]. DATS is conformed to have proliferation effect on human glioblastoma (U87MG) and neuroblastoma (SH SY5Y) cell lines [8,9]. The allicintreated U87MG cells exhibited cell rounding and shrinkage [9]. In vivo, DATS also has the effect of inhibiting cell cycle progression. A study about DATS treatments on ectopic U87MG induced tumors found significant reduction of G2/M phase checkpoint regulator cdk1 and its activator cdc25c, with increased p53 expression. The results show DATS can reduce tumor growth by inhibits cell cycle progression and promotes p53-mediated tumor suppression pathways [10].

\section{Allicin induce apoptosis on GBM}

Inducing apoptosis is a important stage in inhibiting the development and progression of cancer. In this regard, numerous studies based on allicin or its active compounds show effect of inducing apoptosis through different approaches.

Allicin induces apoptosis through intrinsic and extrinsic pathways: The extrinsic death receptor pathway and the intrinsic mitochondrion pathway are the two main apoptosis associated 
signalling pathways. The activation of Fas binding to its Fas ligand (FasL) could initiate the extrinsic pathway of apoptosis and serve a key role involved in death signalling in many cancer types [11]. The intrinsic pathway is mainly regulated by B cell lymphoma 2 (Bcl 2) family proteins which are located on mitochondrial membrane. Bcl-2 family regulate the release of cytochrome $\mathrm{C}$ from the mitochondrial intermembrane space into the cytosol. Cytochrome C then interacts with procaspase- 9 and Apaf- 1 to activate caspase- 9 and then activate caspase-3 leading to apoptosis [12].

A study showed that after treating glioma U251 cells with different amounts of allicin, Fas, FasL and Bax mRNA expression levels were significantly increased, caspase 3, 8 and 9 enzyme activities were upregulated, while $\mathrm{Bcl} 2$ expression levels were decreased, in a dose dependent manner [7]. Another study showed an increase in the Bax: Bcl-2 ratio and mitochondrial release of cytochrome $\mathrm{C}$ and activation of caspase- 9 in glioblastoma cells after treatment with allicin metabolites (DAS, DADS, and DATS) [13]. These results demonstrated that Allicin may induce apoptosis through extrinsic and intrinsic pathways in a dose dependent manner.

Production of ROS induces apoptosis of GBM cells: Evidence suggests that induction of apoptosis by garlic products can be dependent on production of reactive oxygen species (ROS). A significant increase of ROS was induced in A549 lung carcinoma cells after DADS treatment, which may be an early event in DADSmodulated apoptosis [14]. Production of ROS can active some apoptosis inducers which active the mitogen-activated protein kinase (MAPK) pathway further [15]. A study on allicin main metabolites (DAS, DADS, and DATS) found they can increase the ROS production in human glioblastoma T98G cells and U87MG cells and induce p38 MAPK phosphorylation leading to apoptosis. Activation of JNK pathway and $\mathrm{Ca}^{2+}$-dependent pathways are involved in this process [13]. DATS can induce apoptosis by ROS through regulation of $\mathrm{Bcl}-2$ and have anticancer effect on human glioblastoma (U87MG) and neuroblastoma (SH-SY5Y) cells [8]. Another study about the effect of Allicin on U87MG cells also found allicin can induce apoptosis on U87MG cells, and the mechanisms were found to involve the mitochondrial pathway of Bcl-2/Bax, the MAPK/ ERK signalling pathway and antioxidant enzyme systems. The difference is this study shows allicin-induced apoptosis on U87MG is not regulated by the caspase cascade [9].

\section{Inhibition of cancer stem cells(CSC)}

A highly tumorigenic subpopulation of cells found in GBM is responsible for tumor initiation and progression. Because these cells shows stem cell-like properties, such as self-renewal and differentiation into other cell types, these cells are referred to as cancer stem cells (CSC) [16]. The effect of chemotherapy drugs on CSC is an important factor affecting the therapeutic effect on GBM. Yuchae Jung and his colleagues obtained CSC expressing the CD133 marker on the cell surface through culturing human GBM U87 and U251 cells. They found the treatment of ajoene inhibit the sphere forming activity of the two cell lines in a dose-dependent manner. The CD133+ subpopulation in the CSC culture condition was significantly decreased in ajoene-treated cells, and this activity may result from decreased AKT activity, increased ERK/p38 activity, and decreased TGF $\beta$ activity, all of which are associated with downregulated FOXO3A expression [17].

\section{DATS prevents tumor progression and promotes apopto- sis by inhibiting HDAC}

Cancer cells have a common feature of genomic instability are and are more susceptible than normal cells to DNA damaging agents. The DNA damage response is modulated by the acetylation status of histone and non-histone proteins, and by the opposing activities of histone acetyltransferase and histone deacetylase (HDAC) enzymes. HDAC overexpressed in cancer cells has been implicated in protecting such cells from genotoxic insults by activing tumor suppressor genes and DNA repair pathways [18]. Studies on other tumors found DATS can inhibit DNA damage response proteins like p53 by inhibiting HDAC [19]. A study about the effect of DATS on ectopic U87MG induced tumors shows DATS can reduce tumor size and mitosis, decrease HDAC activity, inhibit cell cycle progression, promotes p53-mediated tumor suppression pathways, and increased cell death markers. The findings demonstrate that DATS can inhibit U87MG cell growth in vivo by inhibiting HDAC [10].

\section{Allicin enhances TMZ sensitivity in GBM}

Temozolomide (TMZ) has been the standard chemotherapy for newly diagnosed GBM for many years. Although TMZ can prolong the survival time by a small margin, a considerable number of glioblastoma patients are insensitive to TMZ treatment or gradually develop secondary resistance. Therefore, GBM resistance to TMZ is considered to be the underlying cause of chemotherapy failure and GBM recurrence [20]. Studies have demonstrated that the most important mechanism of TMZ resistance is the expression of 06-methylguanine-DNA methyltransferase (MGMT) [21]. MGMT protein is a kind of DNA repair enzyme, which can protect normal tissues from alkylating agent damage, reduce cancer, and also cause tumor tissue resistance to alkylating agent chemotherapy. High expression of MGMT will lead to TMZ resistance and chemotherapy failure. Acetylation of histones $\mathrm{H} 3$ and $\mathrm{H} 4$ can up regulate the expression of MGMT [22]. Recent study shows allicin can promote apoptosis and enhance the sensitivity of TMZ-resistant U251 cells to TMZ by promoting the high expression of miR-486-3p which can directly bound to the 3'-UTR of MGMT and decreased the protein expression of MGMT through inhibiting the translation of the MGMT mRNA into the MGMT protein [23].

\section{Conclusion}

Allicin is a naturally occurring compound, is consumed by large populations with no ill effects. Previous researches have showed allicin and its metabolites do a vital role in the killing of multiple cancers and the inhibition of the cancer progression. There are only a few studies about the treatment of allicin on GBM. We 
summarized previous studies and found allicin has a therapeutic effect on GBM in multiple ways. Allicin and its metabolites are a range of organic sulfides. They can increase the ROS production and induce apoptosis of GBM cells in vitro and in vivo, and inhibit the activity of CSC. Inhibition of HDAC may be one of the important mechanisms. The most interesting thing is allicin can enhance the sensitivity of TMZ-resistant cells to TMZ by inhibiting MGMT expression. Many mechanisms are still unclear and require further study. Allicin may be an effective drug for clinical GBM treatment and can be generated at low cost to patients.

\section{Acknowledgement}

None.

\section{Conflict of Interest}

Author declare no conflict of interest.

\section{References}

1. Ostrom QT, Gittleman H, Xu J, Kromer C, Wolinsky Y, et al. (2016) CBTRUS statistical report: Primary brain and other central nervous system tumors diagnosed in the United States in 2009-2013. Neuro Oncol 18(suppl_5): v1-v75.

2. Stupp R, Mason WP, van den Bent MJ, Weller M, Fisher B, et al. (2005) Radiotherapy plus concomitant and adjuvant temozolomide for glioblastoma. N Engl J Med 352(10): 987-996.

3. Jennifer C, Nicholas B, Susan C (2010) Recent Advances in Therapy for Glioblastoma. Arch Neurol 67(3): 279-283.

4. Londhe V, Gavasane AT (2011) Role of garlic (Allium sativum) in various diseases: An overview. J Pharmaceu Res Opinion 1: 129-134.

5. Corzo-Martinez M, Corso N, Villamiel M (2007) Biological properties of onions and garlic. Trends Food Sci Technol 18: 609-625.

6. Rana SV, Pal R, Vaiphei K, Sharma SK, Ola RP (2011) Garlic in health and disease. Nutr Res Rev 24(1): 60-71.

7. Li CL, Jing HG, Ma GT, Liang P (2018) Allicin induces apoptosis through activation of both intrinsic and extrinsic pathways in glioma cells. Mol Med Rep 17(4): 5976-5981.

8. Jurkowska H, Wrobel M, Kaczor-Kaminska M, Jasek-Gajda E (2017) A possible mechanism of inhibition of U87MG and SH-SY5Y cancer cell proliferation by diallyl trisulfide and other aspects of its activity. Amino Acids 49(11): 1855-1866.

9. Cha JH, Choi YJ, Cha SH, Choi CH, Cho WH (2012) Allicin inhibits cell growth and induces apoptosis in U87MG human glioblastoma cells through an ERK-dependent pathway. Oncol Rep 28(1): 41-48.
10. Wallace GC, Haar CP, Vandergift WA, Giglio P, Dixon-Mah YN, et al. (2013) Multi-targeted DATS Prevents Tumor Progression and Promotes Apoptosis in an Animal Model of Glioblastoma via HDAC-inhibition. J Neurooncol 114(1): 43-50.

11. Wang W, Zheng Z, Yu W, Lin H, Cui B, et al. (2012) Polymorphisms of the FAS and FASL genes and risk of breast cancer. Oncol Lett 3(3): 625628.

12. Yuan Q, Ray RM, Johnson LR (2000) Polyamine depletion prevents camptothecin-induced apoptosis by inhibiting the release of cytochrome c. Am J Physiol Cell Physiol 282(6): C1290-C1297.

13. Das A, Banik NL, Ray SK (2007) Garlic Compounds Generate Reactive Oxygen Species Leading to Activation of Stress Kinases and Cysteine Proteases for Apoptosis in Human Glioblastoma T98G and U87MG Cells. Cancer 110(5): 1083-1095.

14. Wu XJ, Kassie F, Mersch-Sundermann V (2005) The role of reactive oxygen species (ROS) production on diallyl disulfide (DADS) induced apoptosis and cell cycle arrest in human A549 lung carcinoma cells. Mutat Res 579(1-2): 115-124.

15. Kwon KB, Yoo SJ, Ryu DG, Yang JY, Rho HW, et al. (2002) Induction of apoptosis by diallyl disulfide through activation of caspase-3 in human leukemia HL-60 cells. Biochem Pharmacol 63: 41-47.

16. Hyun KH, Yoon CH, Kim RK, Lim EJ, An S, et al. (2011) Eckol suppresses maintenance of stem ness and malignancies in glioma stem-like cells. Toxicol Appl Pharmacol 254(1): 32-40.

17. Jung Y, Park H, Zhao HY, Jeon R, Ryu JH, et al. (2014) Systemic Approaches Identify a Garlic-Derived Chemical, Z-ajoene, as a Glioblastoma Multi forme Cancer Stem Cell-Specific Targeting Agent. Mol Cells 37(7): 547553.

18. Rajendran P, Ho E, Williams DE, Dashwood RH (2011) Dietary phytochemicals, HDAC inhibition, and DNA damage/repair defects in cancer cells. Clin Epigenetics 3(1): 4.

19. Lea MA, Randolph VM, Lee JE, desBordes C (2001) Induction of histone acetylation in mouse erythroleukemia cells by some organosulfur compounds including allyl isothiocyanate. Int J Cancer 92(6): 784-789.

20. Kang HC, Kim IJ, Park JH, Shin Y, Ku JL, et al. (2004) Identifcation of genes with diferential expression in acquired drug-resistant gastric cancer cells using high-density oligonucleotide microarrays. Clin Cancer Res 10(1 Pt 1): 272-284.

21. Wick W, Weller M, van den Bent M, Sanson M, Weiler M, et al. (2014) MGMT testing-the challenges for biomarker-based glioma treatment. Nat Rev Neurol 10(7): 372-385

22. Nakagawachi T, Soejima H, Urano T, Zhao W, Higashimoto $\mathrm{K}$, et al. (2003) Silencing effect of CpG island hypermethylation and histone modifications on 06-methylguanine-DNA methyltransferase (MGMT) gene expression in human cancer. Oncogene 22(55): 8835-8844.

23. Wu HG, Li X, Zhang T, Zhang GJ, Chen J, et al. (2020) Overexpression miR-486-3p Promoted by Allicin Enhances Temozolomide Sensitivity in Glioblastoma Via Targeting MGMT. Neuro Molecular Medicine. 